\title{
Hydration structure analysis of lysozyme amyloid fibrils by thermally stimulated depolarization currents (TSDC) technique
}

\author{
Maria Grazia Bridelli* and Rosanna Capelletti \\ Dipartimento di Fisica and CNISM, University of Parma, Parma, Italy
}

\begin{abstract}
Thermally stimulated depolarization currents technique has been employed to investigate the conformation of hen egg white lysozyme in native and amyloid form, in the state of powder at very low hydration level. The technique, able to detect the current generated by thermally activated reorientation of water dipoles previously oriented by an electric field, exploits $\mathrm{H}_{2} \mathrm{O}$ dipoles, belonging to the solvation shell, as a probe to gain information on the protein conformation.

Large differences are detected between the TSDC spectra related to the two different protein conformations, for what concerns the number and position of the main peaks, the native form displaying two peaks, at $T_{\mathrm{M}}=175 \mathrm{~K}$ and at $T_{\mathrm{M}}=297 \mathrm{~K}$, and the amyloid one, only one at intermediate temperature $\left(T_{\mathrm{M}}=235 \mathrm{~K}\right)$. The spectra have been compared with those monitored for poly-L-lysine (MW 80400), as received and prepared in different ways, i.e. $\alpha$-helix, $\beta$-sheet, and coil conformation, respectively. The poly-L-lysine spectra show specific features that can be attributed to water texture around the secondary structure adopted by the macromolecule: the results stress how TSDC technique is a tool of great potential value in the conformational analysis of proteins.
\end{abstract}

Keywords: Thermally stimulated depolarization currents, hydration structure, water dipoles, secondary structure, amyloid fibrils, lysozyme, poly-L-lysine

\section{Introduction}

It is well known that protein molecules are surrounded in the cell by a hydration shell formed by interacting water molecules. The shell is not a continuous network of water molecules but is made up of patches locally distributed in dependence of the heterogeneous surface chemistry or occluded in the internal cavities. They are oriented by the surface properties of the protein and are named "bound" molecules, due to the restricted motions with respect to water molecules in pure bulk: ordering changes may indicate structural or conformational differences among macromolecules. The bound water limited mobility allows molecule reorientation, and can be described in term of relaxation times. Different relaxation times can be easily distinguished by using the thermally stimulated depolarization currents (TSDC) technique.

TSDC technique was applied in the past to a variety of natural and synthetic biopolymers and has provided a valuable contribution to the understanding of the complex behaviour of the structured wa-

\footnotetext{
*Corresponding author: M.G. Bridelli, Department of Physics, University of Parma, Viale G.P. Usberti 7/A, Parco Area delle Scienze, 43100 Parma, Italy. Tel.: +39 0521 906227; Fax: +39 0521 905223; E-mail: mariagrazia.bridelli@ unipr.it.
} 
ter. Collagen, keratin, lysozyme, bovine serum albumin, casein, melanin, a number of living tissues, and other biological materials have been studied. Different mechanisms, as non-interacting dipole reorientation, Maxwell-Wagner relaxation, and protonic percolation have been envisaged as responsible for the peaks associated to water in a configuration variety. The wide range of applications offered by the technique has been shown by many authors for a lot of systems and objects $[1,3,13]$; a recent comprehensive review summarizes principles and results obtained with TSDC spectroscopy in the last 20 years [9]. The hydration level $(h)$ of the samples, suitable for TSDC measurements, is very low. However, by proper tuning around the $h$ values, which assure the biological functionality ( $h>0.2 \mathrm{~g}$ of water/g of protein) [5], extensive studies of water uptake and organization have been performed thanks to the very high sensitivity of the technique. For proteins, in particular, the analysis of the distribution of water molecule families, differing in the activation energies, in the number of molecules, and in the volume occupied by them in the biopolymer, provided a simplified hydration model [2].

The present work aims to extend earlier studies by investigating the TSDC spectra dependence on the conformational form of proteins. According to a recent study performed on lipase, by means of coupled TSDC and FTIR spectroscopy measurements, TSDC spectra show changes which are strongly related to the polypeptide conformational changes as a function of the sample hydration [1]. This result, which could not be explained in detail due to a variety of overlapping effects occurring in a complex system such as a protein, is resumed in the present work. Our goal is to understand how water structure is affected in the immediate environment of the protein by the protein surface conformation and how such differences may be monitored by the TSDC spectrum. Therefore our attention was focused on the choice of the samples. Hen egg white lysozyme (HEWL) is a well known protein, already studied in detail even by means TSDC technique. Its secondary structure can be unfolded in relatively simple way, following procedures largely tested and available in the literature. Simple polypeptide such as poly-L-lysine (PL) has been chosen as a model system: in fact, its secondary structure can be properly modified and the water molecules have been employed as probes.

The work has been structured in two phases. At first, TSDC spectra are measured on lysozyme samples either prepared in the native form or in the amyloid conformation. HEWL is an $\alpha+\beta$ protein with $30 \%$ helical residues and $13 \% \beta$-sheet content [11]. Incubation of the protein at $\mathrm{pH} 2.0$ and $45^{\circ} \mathrm{C}$ results in an amyloidogenic form with a predominance of $\beta$ structure [12]. Fourier transform infrared (FTIR) spectroscopy and far ultraviolet circular dichroism (UV-CD) measurements were employed to control some steps along the samples preparation procedure, as described in the Experimental section. In a second phase the TSDC spectra were monitored on a simple model system such as poly-L-lysine: the samples employed were "as received" from suppliers, prepared in $\alpha$-helix, $\beta$-sheet, and coil form. PL is a very flexible system, frequently used to study protein conformation, because it allows preparing samples extensively characterized by one of the three common secondary structures. In fact, at neutral $\mathrm{pH}$ it exhibits a random coil conformation, at alkaline $\mathrm{pH}$ it undergoes the transition to the $\alpha$-helical form, and it shows the $\beta$-structure upon heating in the $30-40^{\circ} \mathrm{C}$ range.

The TSDC spectra for the different samples have been analyzed and compared to gather information on the structural organization of water around the polypeptide chains arranged in different conformations. 


\section{Materials and methods}

\subsection{Samples preparation}

Lysozyme from hen egg white (HEWL, MW 14600) was purchased from SIGMA and used without any further chemical purification. In order to generate amyloid fibrils the protein was incubated at $\mathrm{pH} 2.0$ and $45^{\circ} \mathrm{C}$ for 2 weeks following the procedure described in [12]. After several days of incubation, a gelification-like process occurred and the unfolding of the tertiary structure was monitored by means of FTIR and CD techniques. After 15 days of incubation the sample was dried in air in the oven at $45^{\circ} \mathrm{C}$.

Poly-L-lysine (PL) hydrobromide (MW 80400) was purchased from SIGMA, purified by dialysis against $0.01 \mathrm{M} \mathrm{HCl}$, and lyophilized. The powder obtained was dissolved in $0.2 \mathrm{M} \mathrm{NaCl}$ and the final $\mathrm{pH}$ was in the neutrality range. At neutral $\mathrm{pH}, \mathrm{PL}$ has a random coil conformation. A part of this solution was titrated to alkaline $\mathrm{pH}$ ( $\mathrm{pH}$ 11) by injecting small aliquots of $1.0 \mathrm{M} \mathrm{NaOH}$, to generate the transition to the $\alpha$-helical conformation. A part of this last sample was heated at $40^{\circ} \mathrm{C}$ for one hour to induce the formation of the $\beta$-structure. The first two types of samples were allowed to dry in air at room temperature to preserve as far as possible their conformation, while the third one was dried in air in a oven at $40^{\circ} \mathrm{C}$ to avoid a $\beta-\alpha$ interconversion, which might occur by lowering the temperature [7].

All the samples were powdered, maintained in air, and not sealed, before submitting them to any measurement. The estimated air humidity was about $70 \%$.

\subsubsection{FTIR measurements}

The amide I region in the infrared spectra of polypeptides is very sensitive to their secondary structure. Several methods of analysis can be applied to predict the protein conformation, by decomposing the amide I band into individual components, corresponding to the different types of secondary structure [6]. Thus FTIR spectroscopy has been therefore chosen to control the secondary structure content. The samples were thin films obtained by depositing aqueous solutions $(c=10 \mathrm{mg} / \mathrm{ml})$ on $\mathrm{CaF}_{2}$ windows and allowing them to dry in air. The absorption spectra were collected with a Jasco 420 FTIR spectrophotometer operating in the $4000-600 \mathrm{~cm}^{-1}$ range, at temperature close to ambient (290-300 K). Each spectrum was measured by acquiring $128 \mathrm{scans}$ at $2 \mathrm{~cm}^{-1}$ spectral resolution. Fitting of the amide bands was performed according to Gaussian curves starting from a second derivative analysis. Origin 7.0 software was used for the absorption band deconvolution.

\subsubsection{CD measurements}

Circular dichroism spectra were recorded by using a Jasco J715 spectropolarimeter. Far-UV (185$250 \mathrm{~nm})$ spectra were recorded on aqueous solution $(c=0.5 \mathrm{mg} / \mathrm{ml})$ in $0.01 \mathrm{~cm}$ path length cell, using a step size of $0.5 \mathrm{~nm}$, bandwidth of $1 \mathrm{~nm}$, and scan rate of $20 \mathrm{~nm} / \mathrm{min}$. Each protein spectrum was obtained by averaging 3 scans and was corrected by subtracting the solvent spectrum.

\subsubsection{TSDC measurements}

The samples for TSDC measurements were pellets of $13 \mathrm{~mm}$ diameter and thickness ranging between 0.20 and $0.35 \mathrm{~mm}$, prepared by applying a pressure of $7.4 \times 10^{8} \mathrm{~Pa}$ to $50 \mathrm{mg}$ of powdered material. The pellet was then placed between stainless steel electrodes. A standard apparatus was used [4] and the spectra were recorded in the temperature range 100-300 K. An electric field $E_{\mathrm{p}}$ in the $(0.25-2) \times$ $10^{4} \mathrm{~V} / \mathrm{cm}$ range was applied to the sample for a polarization time $t_{\mathrm{a}} \sim 3 \mathrm{~min}$. The heating rate was about $0.15 \mathrm{~K} / \mathrm{s}$. To minimize dehydration of the sample, TSDC measurements were performed in dry nitrogen atmosphere at a pressure of $1.33 \times 10^{4} \mathrm{~Pa}$. Between two subsequent TSDC runs the nitrogen pressure was increased to $8 \times 10^{4} \mathrm{~Pa}$. 


\subsection{TSDC technique}

TSDC is the current generated by the thermally activated release of stored dielectric polarization during controlled heating [4]. The sample, in form of pellet or powder, is placed in a closed vessel, where vacuum or a controlled gaseous atmosphere is established. The sample is sandwiched between metal electrodes (in general, with plane symmetry). The measurement consists in three steps:

(1) the sample is polarized by a strong dc electric field $E_{\mathrm{p}}$ at a temperature $T_{\mathrm{p}}$;

(2) the polarization is "frozen in" by cooling down to a sufficiently low temperature $T_{\mathrm{f}}$ at which the dipoles and the charges are no longer mobile: the electric field is then removed;

(3) the displacement current $J$ is monitored during the subsequent heating as a function of the temperature $T$ (TSDC spectrum). During this step the dipoles gain mobility and recover the initial random distribution, originating the TSDC signal.

For non-interacting dipole reorientation, characterized by monomolecular kinetics, the recorded current $J$ is

$$
J\left(T_{j}\right)=\sum_{i=1}^{N} \frac{P_{0 i}}{\tau_{i}\left(T_{j}\right)} \exp \left(-\frac{1}{b} \int_{T_{0}}^{T_{j}} \frac{\mathrm{d} T^{\prime}}{\tau_{i}\left(T^{\prime}\right)}\right),
$$

where $\tau_{i}(T)$ is the relaxation time for each $i$ of the $N$ elementary processes, $P_{0 i}$ is its contribution to the total polarization and $b$ is the heating rate.

The temperature dependence of the relaxation time follows the Arrhenius law, i.e.

$$
\tau(T)=\tau_{0} \exp \left(\frac{E}{k_{\mathrm{B}} T}\right)
$$

where $\tau_{0}$ is a constant, $E$ the activation energy, $k_{\mathrm{B}}$ the Boltzmann's constant, and $T$ the absolute temperature.

\section{Results and discussion}

\subsection{Lysozyme}

TSDC spectrum of native form lysozyme is shown in Fig. 1 (curve a). It was obtained by polarizing the sample in a wide temperature range $\left(T_{\mathrm{p}}=300 \mathrm{~K}, T_{\mathrm{f}}=100 \mathrm{~K}\right)$. The spectrum is similar to those appeared in literature in the past [2,14], in fact it consists of two main peaks: a weak one at $T_{\mathrm{M}}=175 \mathrm{~K}$, which can be labeled low temperature (LT) peak, and a strong one at $T_{\mathrm{M}}=297 \mathrm{~K}$, high temperature (HT) peak. The amplitude of the HT peak was studied vs. the applied electric field strength $E_{\mathrm{p}}$ : the detected linear behavior in the $E_{\mathrm{p}}$ explored range (see Section 2) suggests that dipoles, as water molecules, should be mainly responsible for the TSDC spectra. The dependence of the two peaks amplitude and position on the water content was previously recognized and studied in detail [2] and revealed that both peaks are characterized by a wide distribution of activation energies and relaxation times: this means that there are different classes of water dipoles coordinated by the polypeptide. In particular, LT peak was attributed to water dipoles buried in the shallow cavities of the macromolecular structure and the HT peak to water at the surface of macromolecule, belonging to the external hydration shell. 


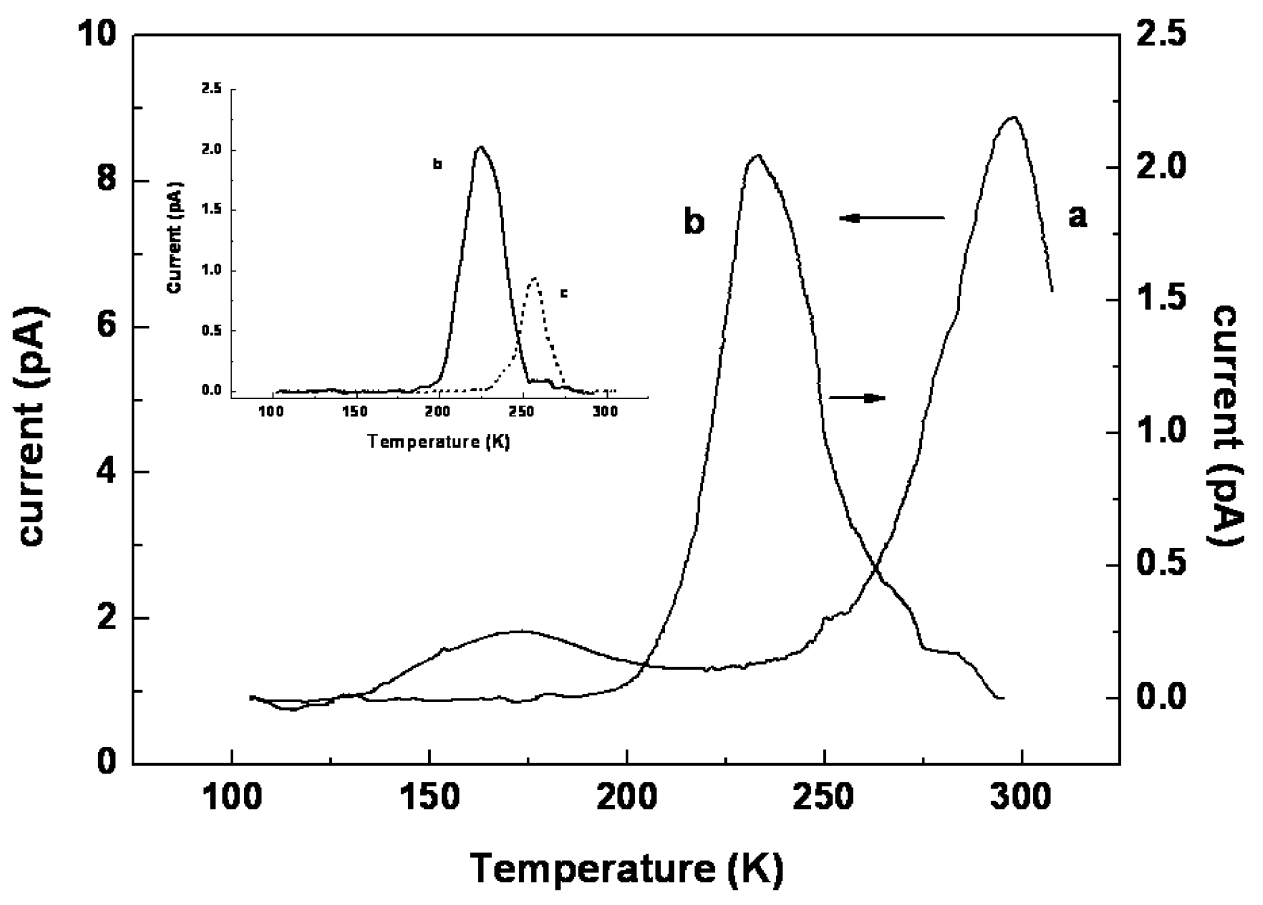

Fig. 1. TSDC spectra of lysozyme in the native (a) and amyloid (b) form $\left(T_{\mathrm{p}}=300 \mathrm{~K} ; T_{\mathrm{f}}=100 \mathrm{~K}\right)$. Inset: TSDC spectra of lysozyme in the amyloid conformation, as prepared (c) and after equilibration in damp air (b). Curves (b) are the same in the two pictures. The samples are pellets of powdered material maintained in air before to submit them to the measurements.

Before monitoring the TSDC spectra of the amyloid protein, FTIR and CD measurements were carried out to verify that the treatment, described in Section 2, has caused indeed the expected conformational changes with the formation of amyloid fibrils. Conformational transition was monitored by the decrease of the FTIR band at $\nu \approx 1654 \mathrm{~cm}^{-1}$, characteristic of $\alpha$-helical structures, and by the progressive increase of the narrow " $\beta$-band" at $\nu \approx 1620 \mathrm{~cm}^{-1}$, as shown in Fig. 2 , where the FTIR spectra of HEWL in the native (a) and unfolded state (b) are compared [15]. Figure 3 monitors the shape changes of the CD spectrum in the $205-222 \mathrm{~nm}$ and the increase of the positive signal at $195 \mathrm{~nm}$, indicating that the dominant structure in the sample at the end of the procedure involves $\beta$-sheet structures [12]. Both measurements show that important changes in the secondary structure have occurred, although the protein is not completely converted into $\beta$ form and both $\alpha$-helical and disordered structures are still surviving.

Previous experimental results [16] point out as changes in the FTIR amide bands are dependent on the physical state of the samples, such as the dried solid state, as a consequence of the hard water removal, in addition to the occurrence of the secondary structure modification. This effect cannot be completely excluded even in the present case, however the dehydration treatment adopted to remove water from the samples, i.e. the evaporation at room or at moderate $\left(40-45^{\circ} \mathrm{C}\right)$ temperature, is milder and slowly progressive with respect to the lyophilization procedure, thus favoring gradual conformational changes.

TSDC spectrum of lysozyme in amyloid form and equilibrated in air for three days (Fig. 1, curve b) shows a single band, peaking in the intermediate temperature range $\left(T_{\mathrm{M}}=235 \mathrm{~K}\right)$ and displaying a very low amplitude with respect to that of the HT peak in the native sample spectrum (curve a, compare also the different ordinate scales for curves $a$ and $b$ ). The low temperature (LT) feature is absent in curve b. No doubt exists about the key role played by water in molding this spectrum, as well: the 


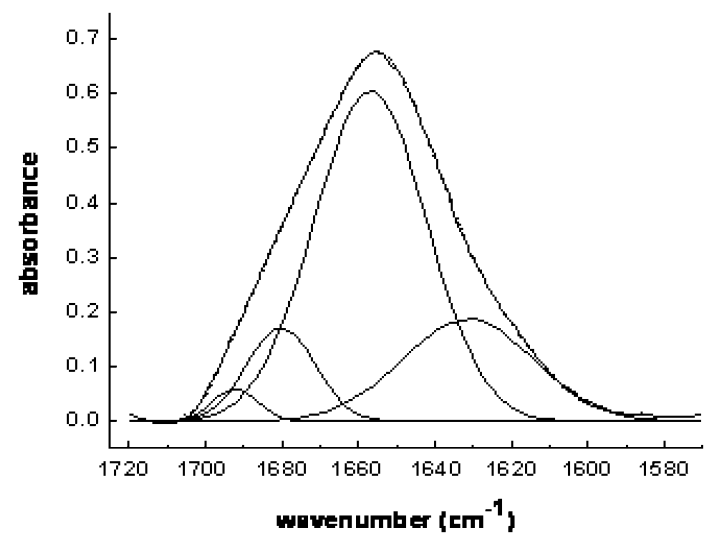

(a)

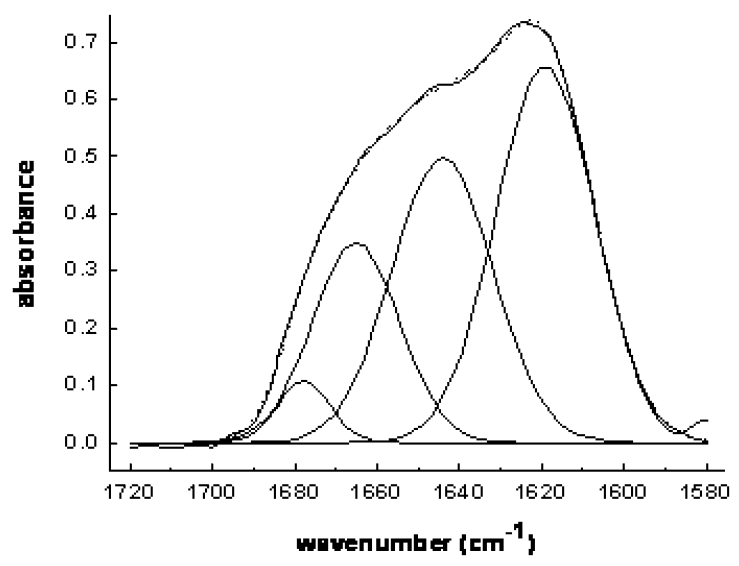

(b)

Fig. 2. Gaussian deconvolution of FTIR spectra in the amide I region for lysozyme in the native (a) and amyloid (b) form, prepared as thin films obtained by evaporation in air at room temperature of a drop of aqueous solution $(c=10 \mathrm{mg} / \mathrm{ml})$ deposited on $\mathrm{a} \mathrm{CaF}_{2}$ windows. The sum of the fitted curves is shown by the thin dotted line, closely overlapping the experimental data trace, which is shown as a full heavy line.

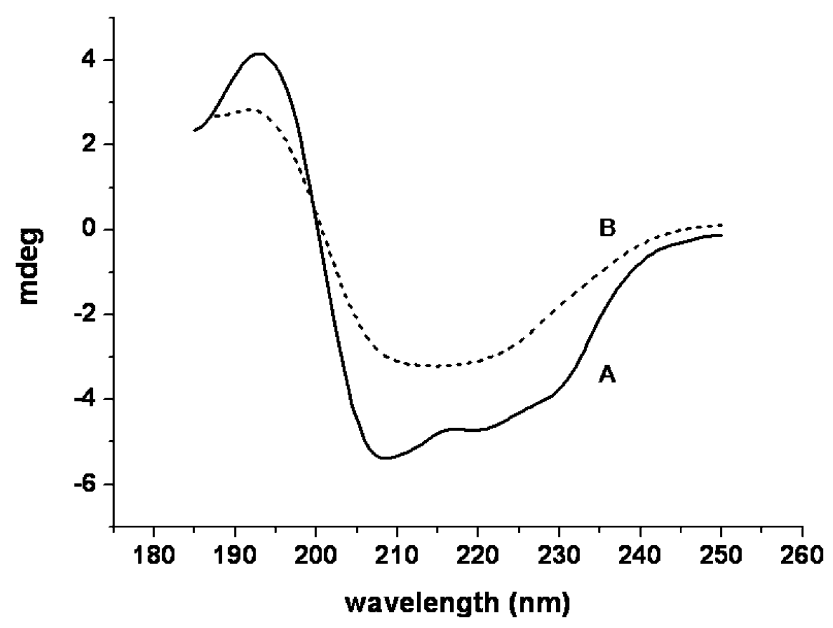

Fig. 3. CD spectra ( $T=297 \mathrm{~K}$ ) of lysozyme (aqueous solution, $c=0.5 \mathrm{mg} / \mathrm{ml}$ ) in the native (A) and amyloid (B) state.

inset compares the above mentioned spectrum (b) (i.e. related to a sample equilibrated in air for three days) with spectrum (c) related to a sample measured immediately after its removal from the oven $\left(T_{\mathrm{M}}=255 \mathrm{~K}\right)$. By increasing the hydration level (curve $\mathrm{b}$ ), the amplitude of the main peak increases with respect to the peak displayed by curve $\mathrm{c}$ and its position shifts towards the low temperature region $\left(T_{\mathrm{M}}=235 \mathrm{~K}\right)$, while the LT peak does not recover at all. The shift of the main peak in the spectrum of the amyloid sample with respect to the native one, for samples at the same hydration content, might be easily related to the changes of the macromolecule surface coordinating the surrounding layer of hydrating water molecules. The reduced $235 \mathrm{~K}$ band amplitude and the lacking of the LT peak deserve an additional comment. The fibrillar conformation presumably offers a significantly lower number of coordination sites for water molecules, both on the surface and in the interior, than the folded form. This can be explained by the close packing of the $\beta$-sheet segments forming the amyloid structures, where so tightly bundled aggregates are not accessible to the water molecules [8]. 


\subsection{Poly(L-lysine)}

In order to better understand the TSDC spectra of lysozyme, TSDC measurements have been carried out on a simpler model system, such as the polyaminoacid poly-L-lysine. In Fig. 4 the spectrum of the "as received" homopolyaminoacid is displayed. The spectrum shows a very complex structure revealing two main features in the low temperature region $(T<250 \mathrm{~K})$, a magnification of which is portrayed in the inset, at $T_{\mathrm{M}}=160$ and $200 \mathrm{~K}$, respectively, followed by a sharp rise for $T>250 \mathrm{~K}$. In the inset the result of the so called "windowing" technique [17] shows some main individual component peaks, obtained by carefully choosing the polarization range $\left(T_{\mathrm{p}}, T_{\mathrm{f}}\right)$. Figure 5 compares the spectra of the samples "as received" (curve a), and submitted to a drastic dehydration treatment, pursued by exposing the specimen to the dynamical vacuum at room temperature (curve b). The sample annealing in vacuum causes a dramatic spectrum change, once more confirming the key role played by hydration on the TSDC spectrum. The curve b signal is so strongly reduced that it is difficult to resolve the peaks from the background. In the inset the magnification of the spectra is presented, in order to follow in detail the evolution of the main bands as a consequence of dehydration: in the low temperature region the peak amplitude around $T=160 \mathrm{~K}$ decreases, while $200 \mathrm{~K}$ peak shifts towards higher temperatures, without a meaningful amplitude change (within the experimental error). This finding could be explained on the basis of a variety of water coordinations in the interior of the polypeptide folded structure that can be only partially deprived (as a consequence of the annealing treatment) due to their depth or inaccessibility. The high temperature feature is instead strongly suppressed, revealing that the drying process involves the removal of the surface water molecules.

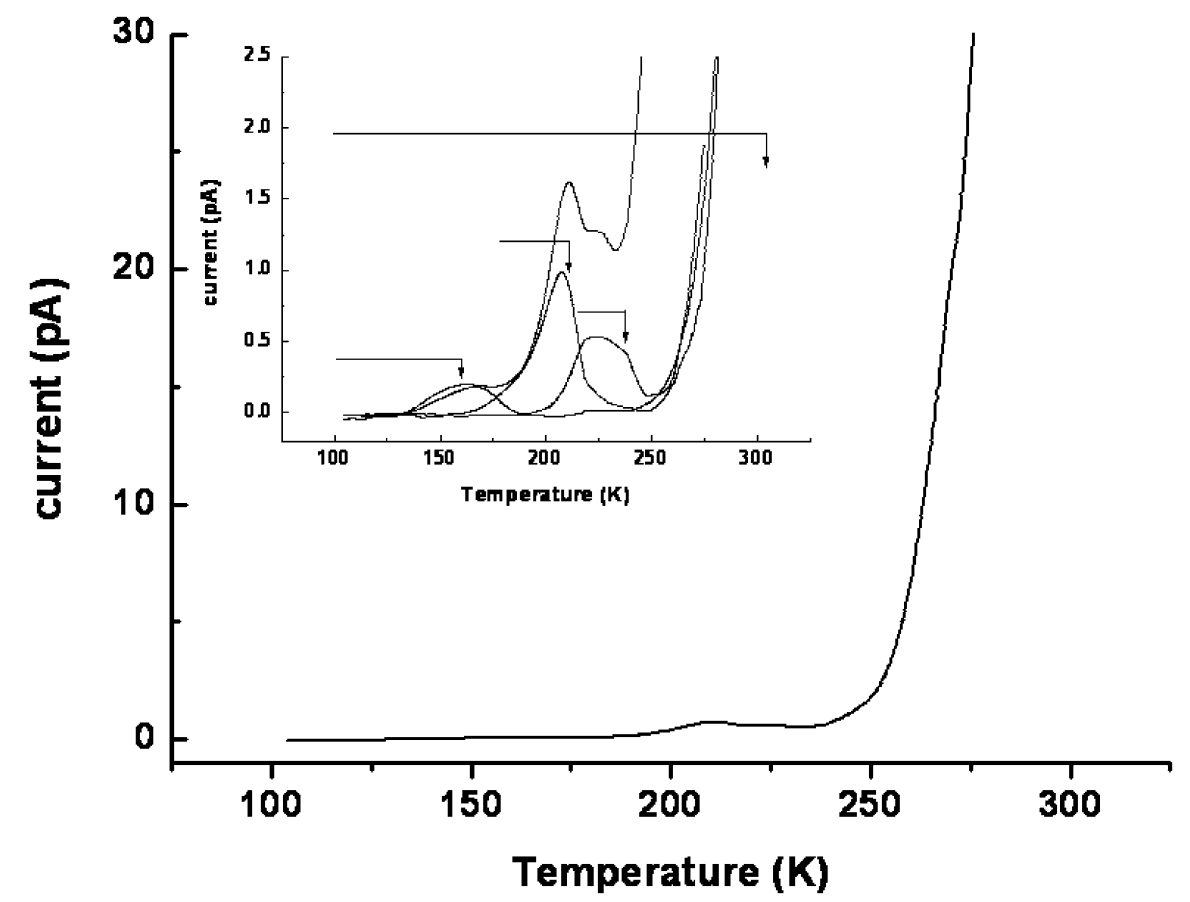

Fig. 4. TSDC spectrum of "as received" poly-L-lysine $\left(T_{\mathrm{p}}=300 \mathrm{~K} ; T_{\mathrm{f}}=100 \mathrm{~K}\right)$. The samples are pellets of powdered material maintained in air before to submit them to the measurements. The inset provides a magnification of the spectrum and shows three component bands resolved by choosing suitable polarization ranges, indicated by arrows and horizontal segments. 


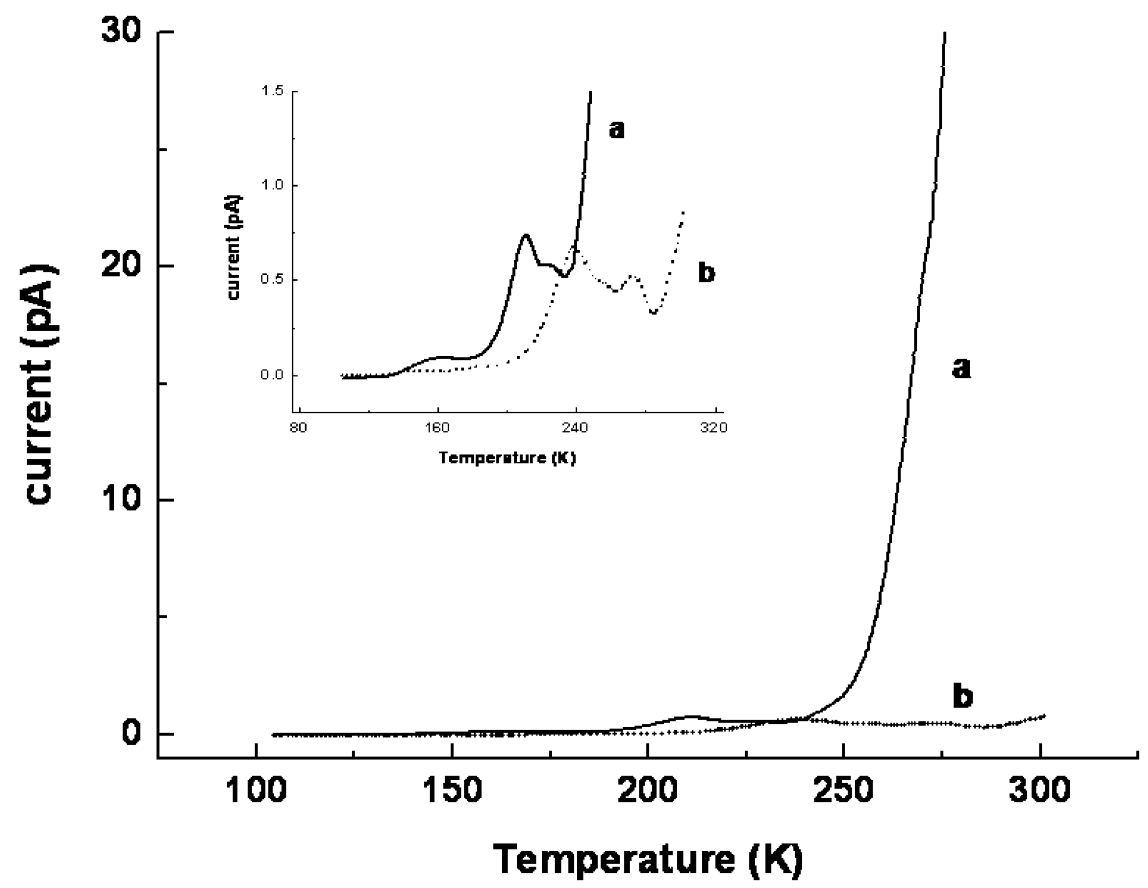

Fig. 5. TSDC spectra of poly-L-lysine pellets "as received" (a curve) and after dehydration treatment in dynamic vacuum for $144 \mathrm{~h}$ at $\mathrm{RT}$ (b curve) $\left(T_{\mathrm{p}}=300 \mathrm{~K} ; T_{\mathrm{f}}=100 \mathrm{~K}\right)$.

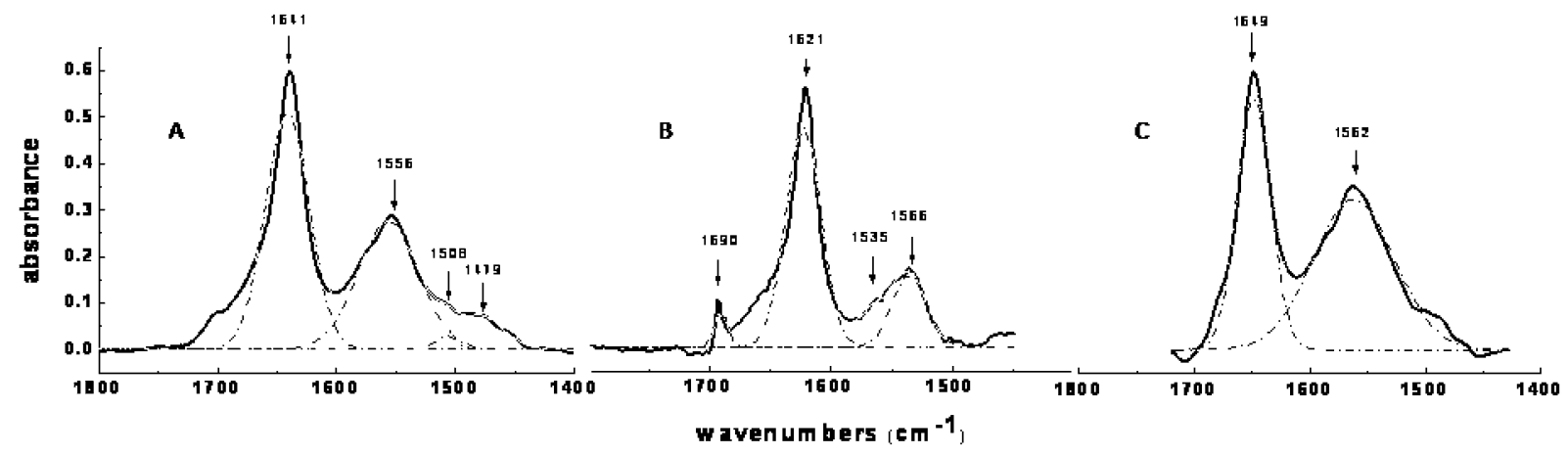

Fig. 6. FTIR spectra in the amide I and II region for poly-L-lysine films (starting aqueous solution $c=10 \mathrm{mg} / \mathrm{ml}$ ) in the $\alpha$-helix (A), $\beta$-sheet (B), and coil (C) conformations. A and C samples were allowed to dry in air at room temperature, B sample was dried in air at $40^{\circ} \mathrm{C}$.

The conformational contributions to TSDC spectra by each ordered element of secondary structure, presumably retained in different percentages in the examined sample, have been isolated and investigated by preparing PL in $\alpha, \beta$ and coil conformation. FTIR spectroscopy has been used to monitor the predominance of each conformational structure: the amide I and II regions for the three samples were investigated. The results are reported in Fig. 6. Shape and band parameters were found in good agreement with the literature data $[10,18]$.

Figure 7 portraits the TSDC spectra of $\alpha$-, $\beta$ - and coil-PL pellets, obtained by polarizing the samples in the temperature range $T_{\mathrm{p}}=300 \mathrm{~K}, T_{\mathrm{f}}=100 \mathrm{~K}$. The three spectra exhibit different shapes, amplitudes, and peak positions. In the $\alpha$-PL spectrum the peak at $T_{\mathrm{M}}=245 \mathrm{~K}$ displays an asymmetrical shape, with 


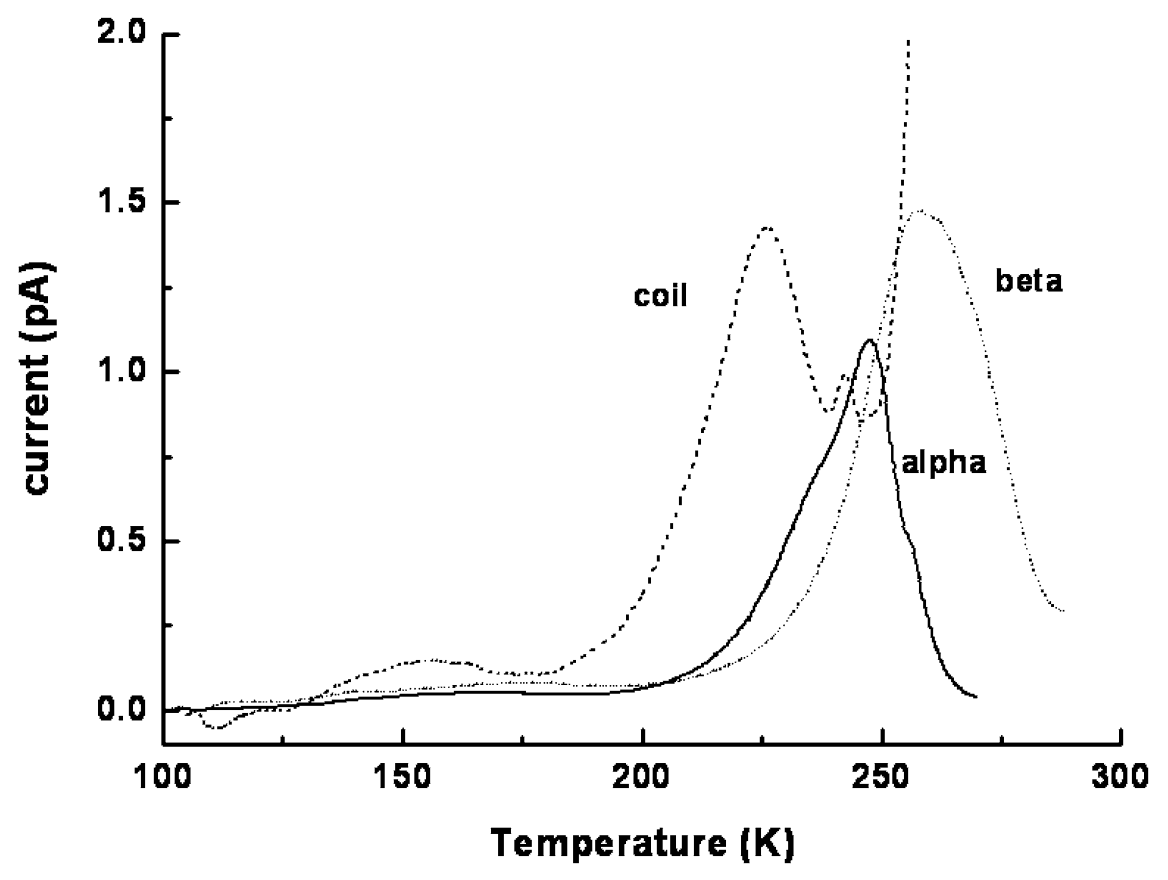

Fig. 7. TSDC spectra of poly-L-lysine in the $\alpha$-helix, $\beta$-sheet, and coil conformations $\left(T_{\mathrm{p}}=300 \mathrm{~K} ; T_{\mathrm{f}}=100 \mathrm{~K}\right)$.

a shoulder around $T=235 \mathrm{~K}$, suggesting that it cannot be attributed to a single relaxation process; $\beta$-PL spectrum exhibits a large band at $T_{\mathrm{M}}=258 \mathrm{~K}$; coil-PL is characterized by complex spectrum, where several component bands can be envisaged, at $T_{\mathrm{M}}=150 \mathrm{~K}, T_{\mathrm{M}}=225 \mathrm{~K}$ and, a weak one at $T_{\mathrm{M}}=242 \mathrm{~K}$, followed by a huge rise for $T>250 \mathrm{~K}$. Such a rise overlaps to the $\alpha$ - and $\beta$-PL spectra, as it is expected, because the two ordered structures surely contribute, although to different extent, to the disordered one. Interestingly, only the disordered biopolymer displays a LT peak, a result which can be related to its folded and, perhaps, flexible conformation, which favors the formation of cavities or crevices where ice-like coordinated water molecules can be hosted.

To understand the role played by each structure on the PL and lysozyme TSDC, the spectra of $\alpha-, \beta-$ and coil-PL are superimposed to those of "as received" poly-L-lysine (Fig. 8), of lysozyme in the native and amyloid forms (Figs 9 and 10, respectively). Although the comparison might induce to put forward a lot of attractive hypotheses, the preliminary character of the measurements suggests some caution in the result interpretation. However, some general considerations are possible. PL spectra, of the "as received" sample before and after the dehydration treatment, are shown in Fig. 8 where a magnification of the low and high temperature region is supplied (panels A and B, respectively). Both the "as received" and dehydrated PL spectra (curves a and b) resemble the coil-PL spectrum: the differences in the peak positions and amplitudes may be attributed to the different percentages by which the secondary structure sequences coordinating water dipoles occur. Some water molecules are structured in the internal loops of the biopolymer and contribute to the LT peak. Vacuum annealing induces a drastic dehydration and a consequent dramatic reduction of the TSDC signal.

A similar comparison applied to lysozyme spectra points out a pronounced resemblance of the native protein spectrum to the PL-coil, at least in the low and high temperature range (Fig. 9), stressing the hypothesis that the main contribution to the signal may originate from the reorientation of water dipoles organized around and into the complex three dimensional structure of the protein containing a variety 


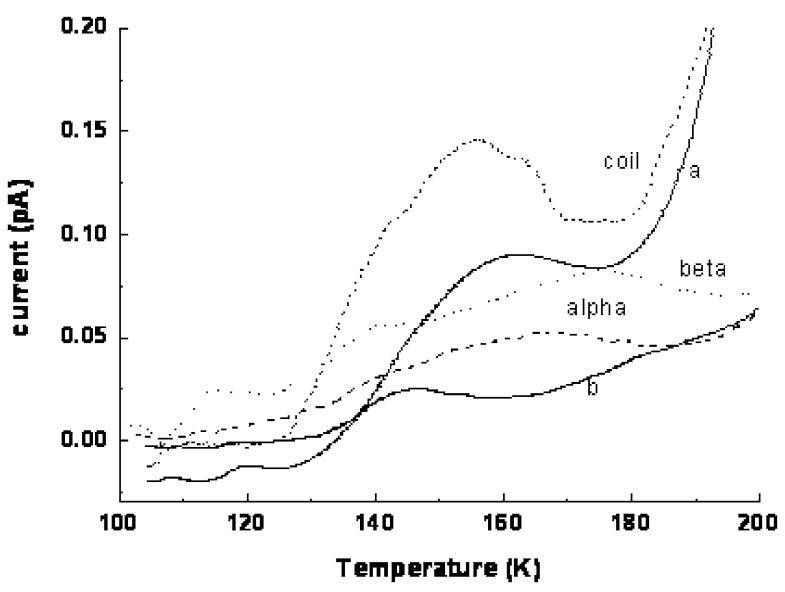

(A)

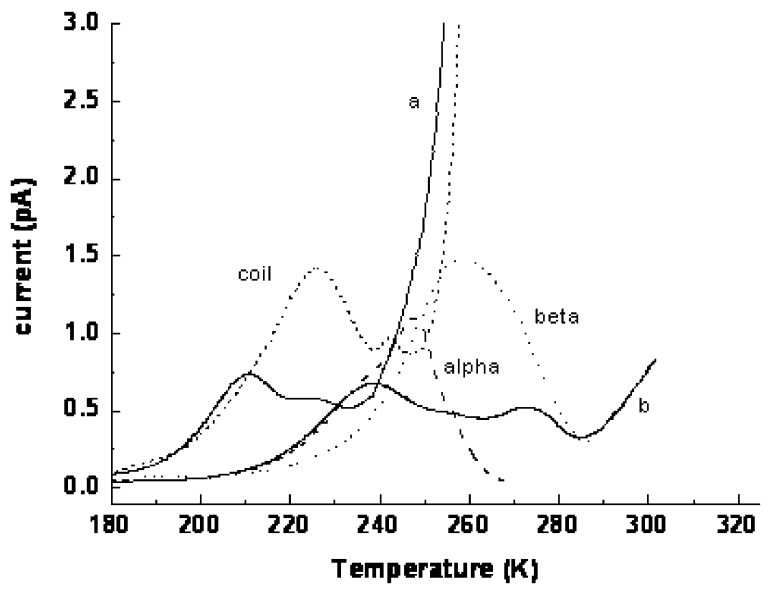

(B)

Fig. 8. TSDC spectra of poly-L-lysine in the $\alpha$-helix, $\beta$-sheet, and coil conformations $\left(T_{\mathrm{p}}=300 \mathrm{~K} ; T_{\mathrm{f}}=100 \mathrm{~K}\right)$ compared with the TSDC spectra of poly-L-lysine "as received" (a) and in the dehydrated state (b). Panel (A): detail of the LT region. Panel (B): detail of the HT region.

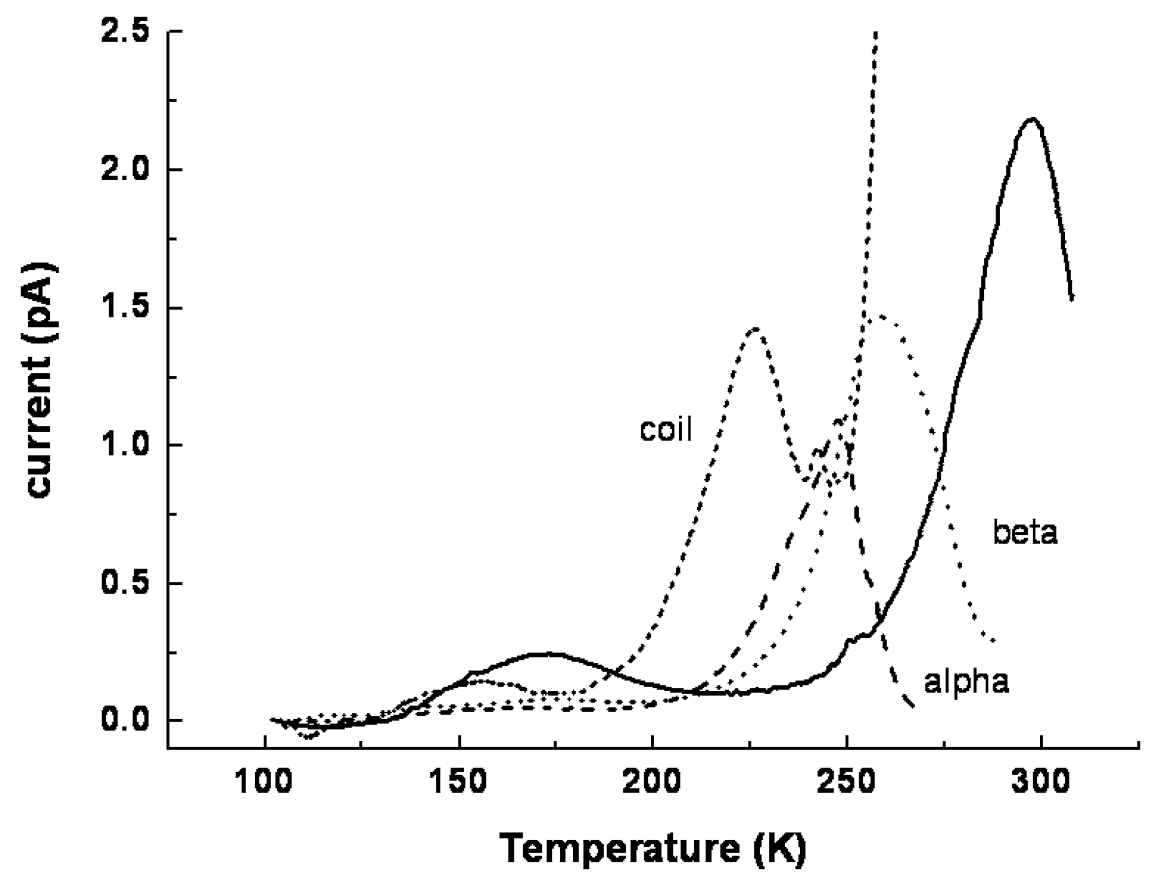

Fig. 9. TSDC spectra of poly-L-lysine in the $\alpha$-helix, $\beta$-sheet, and coil conformations $\left(T_{\mathrm{p}}=300 \mathrm{~K} ; T_{\mathrm{f}}=100 \mathrm{~K}\right)$ compared with the TSDC spectrum of lysozyme in the native form.

of domains, characterized by different secondary structures. This finding could explain also the similar trend exhibited by the TSDC spectra of the different proteins studied in the past.

The $\alpha$-PL and $\beta$-PL spectra work as reference spectra for analyzing those of lysozyme in the amyloid form. All three secondary structures seem to contribute to the TSDC band at $235 \mathrm{~K}$, however the helical 


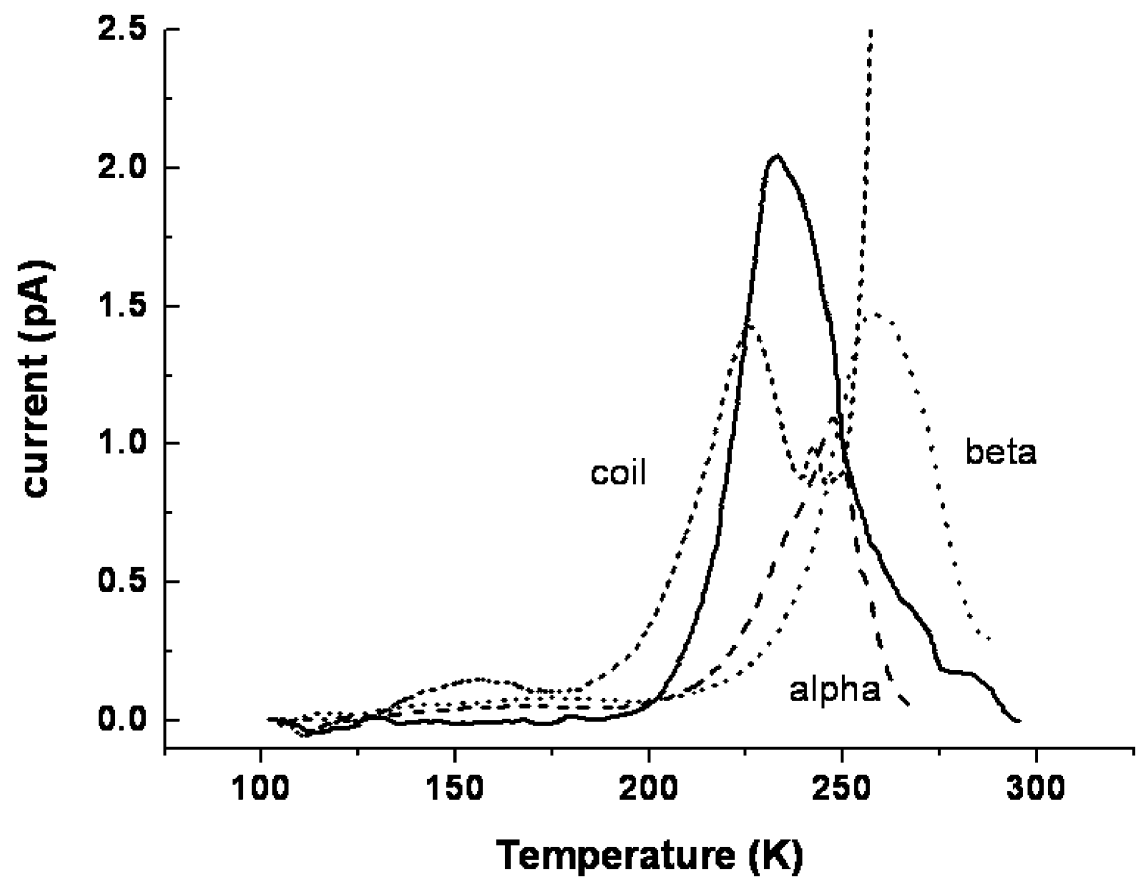

Fig. 10. TSDC spectra of poly-L-lysine in the $\alpha$-helix, $\beta$-sheet, and coil conformations $\left(T_{\mathrm{p}}=300 \mathrm{~K} ; T_{\mathrm{f}}=100 \mathrm{~K}\right)$ compared with the TSDC spectrum of lysozyme in the amyloid form.

one prevails. This result is not in contrast with the expected dominance of the $\beta$-structure, as described in the literature and predicted by FTIR and CD measurements, as well. In fact, the lysozyme TSDC spectrum displayed in Fig. 10 is the same of curves b in Fig. 1, i.e. the sample has been equilibrated in damp air after the incubation at $40^{\circ} \mathrm{C}$ (see Section 2). The cooling and hydrating procedures might have promoted a partial interconversion $\beta-\alpha$, and the consequent shift of the TSDC peak position. In fact, if the spectrum of the "as prepared" sample (inset of Fig. 1, curve c) is compared to the elementary PL spectra, the $\beta$-structure appears to prevail in the amyloid lysozyme. The absence of the low temperature feature can be explained by the lacking of water molecules trapped in the macromolecule interior and corroborates the picture of the amyloid protein, i.e. the fibers are so tightly packed that the inner interstices are no longer accessible to the solvent.

In conclusion TSDC technique, proven in the past to be a non-conventional tool for studying the complex behavior of water molecules bound to biological macromolecules, is confirmed to be particularly valuable for analyzing the conformational conversion of a protein. It has allowed, in fact, recognizing in the lysozyme TSDC spectra the main features of the hydration as a function of secondary structure, although no quantitative evaluation of the protein secondary structure has been achieved in the present work.

Nevertheless, the present preliminary approach opens new perspectives in the future application of the technique for the quantitative analysis of the secondary structure of proteins. Detailed TSDC studies, coupled with the knowledge of the structure, might allow building for a large number of proteins a set of reference spectra to which compare and on which calibrate the TSDC signal recorded on unknown polypeptides. This approach could considerably increase the information content offered by TSDC spectra and unveil a lot of mechanisms involving structural protein modification. 


\section{Acknowledgements}

The authors are grateful to Dr. Roberta Bedotti for assistance in the sample preparation and for CD spectra recording.

\section{References}

[1] M.G. Bridelli, R. Capelletti, F. Maraia, C. Mora and L. Pirola, Initial hydration steps in lipase studied by means of water sorption isotherms, FTIR spectroscopy and thermally stimulated depolarization currents, J. Phys. D: Appl. Phys. 35 (2002), 1039-1048.

[2] M.G. Bridelli, R. Capelletti and A. Vecli, Sequential hydration-dehydration studies of lysozyme by the thermally stimulated depolarization currents (TSDC) technique, J. Biochem. Biophys. Methods 24 (1992), 135-146.

[3] R. Capelletti and M.G. Bridelli, TSDC as a tool to monitor the electret state induced by water in biomolecules, in: ISE10 Proceedings, A.A. Konsta, A. Vassilikou-Dova and K. Vartzeli-Nikaki, eds, IEEE Service Center, New York, 1999, pp. 159-166.

[4] R. Capelletti and R. Fieschi, in: Electrets, M.M. Perlman ed., The Electrochemical Society, Princeton, NY, 1972, pp. 1-10.

[5] G. Careri, E. Gratton, P.H. Yang and J.A. Rupley, Correlation of IR spectroscopic, heat capacity, diamagnetic susceptibility and enzymatic measurements on lysozyme powder, Nature 284 (1980), 572-573.

[6] F. Dousseau and M. Pezolet, Determination of the secondary structure content of proteins in aqueous solutions from their amide I and amide II infrared bands. Comparison between classical and partial least-squares methods, Biochemistry 29 (1990), 8771-8779.

[7] W. Dzwolak, T. Muraki, M. Kato and Y. Taniguchi, Chain length dependence of $\alpha$-helix to $\beta$-sheet transition in polylysine: model of protein aggregation studied by temperature-tuned FTIR spectroscopy, Biopolymers 73 (2004), 463-469.

[8] E. Frare, M.F. Mossuto, P. Polverino de Laureto, M. Dumoulin, C.M. Dobson and A. Fontana, Identification of the core structure of lysozyme amyloid fibrils by proteolysis, J. Mol. Biol. 361 (2006), 551-561.

[9] V.M. Gun'ko, V.I. Zarko, E.V. Goncharuk, L.S. Andriyko, V.V. Turov, Y.M. Nychiporuk, R. Leboda, J. SkubiszewskaZieba, A.L. Gabchak, V.D. Osovskii, Y.G. Ptushinskii, G.R. Yurchenko, O.A. Mishchuk, P.P. Gorbik, P. Pissis and J.P. Blitz, TSDC spectroscopy of relaxational and interfacial phenomena, Adv. Coll. Interf. Sci. 131 (2007), 1-89.

[10] M. Jackson, P.I. Haris and D. Chapman, Conformational transitions in poly(L-lysine): studies using Fourier transform infrared spectroscopy, Biochim. Biophys. Acta 998 (1989), 75-79.

[11] T. Knubovets, J.J. Osterhout, P.J. Connolly and A.M. Klibanov, Structure, thermostability and conformational flexibility of hen egg-white lysozyme dissolved in glycerol, Proc. Natl. Acad. Sci. USA 96 (1999), 1262-1267.

[12] L.A. Morozova-Roche, J. Zurdo, A. Spencer, W. Noppe, V. Receveur, D.B. Archer, M. Joniau and C.M. Dobson, Amyloid fibril formation and seeding by wild-type human lysozyme and its diseases-related mutational variants, J. Struct. Biol. 130 (2000), 339-351

[13] P. Pissis, Dielectric studies of protein hydration, J. Mol. Liq. 41 (1989), 271-289.

[14] P. Pissis and A. Anagnostopoulou-Konsta, Protonic percolation on hydrated lysozyme powders studied by the method of thermally stimulated depolarization currents, J. Phys. D: Appl. Phys. 23 (1990), 932-939.

[15] W.K. Surewicz, H.H. Mantsch and D. Chapman, Determination of protein secondary structure by Fourier transform infrared spectroscopy: a critical assessment, Biochemistry 32 (1993), 389-394.

[16] M. van de Weert, P.I. Haris, W.E. Hennink and D.J.A. Crommelin, Fourier transform infrared spectrometric analysis of protein conformation: effect of sampling method and stress factors, Anal. Biochem. 297 (2001), 160-169.

[17] J. van Turnout, Topics in applied physics, in: Electrets, G.M. Sessler, ed., Springer, Berlin, 1980, pp. 81-215.

[18] S.Yu. Venyaminov and N.N. Kanin, Quantitative IR spectrophotometry of peptide compounds in water $\left(\mathrm{H}_{2} \mathrm{O}\right)$ solutions. II. Amide absorption bands of polypeptides and fibrous proteins in $\alpha-, \beta$-, and random coil conformation, Biopolymers 30 (1990), 1259-1271. 


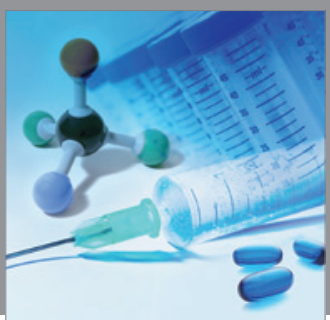

International Journal of

Medicinal Chemistry

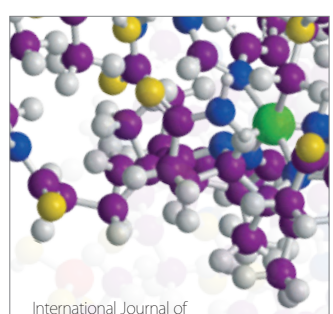

Carbohydrate Chemistry

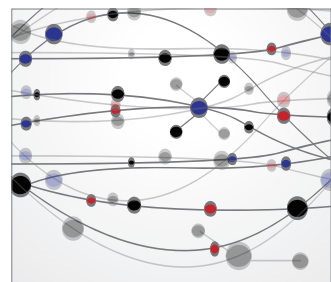

The Scientific World Journal
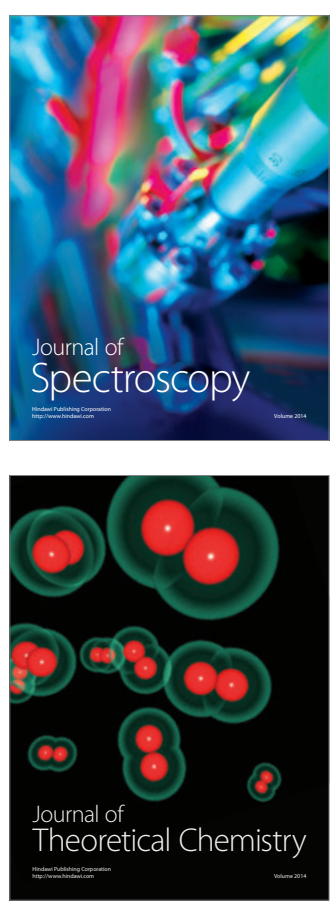
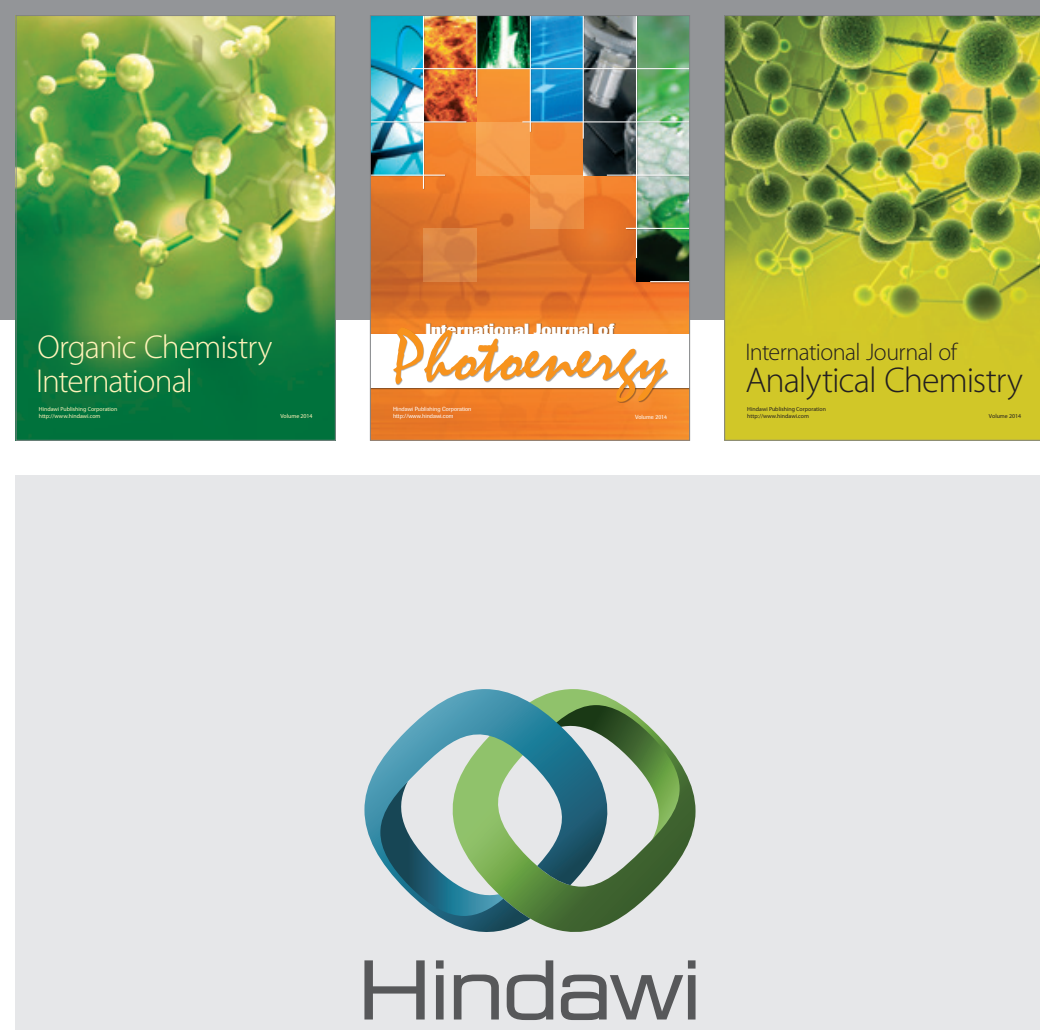

Submit your manuscripts at

http://www.hindawi.com
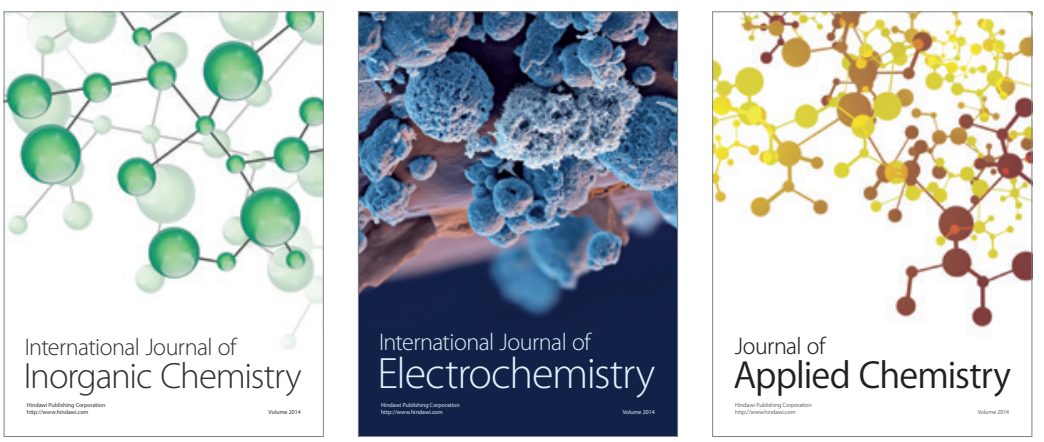

Journal of

Applied Chemistry
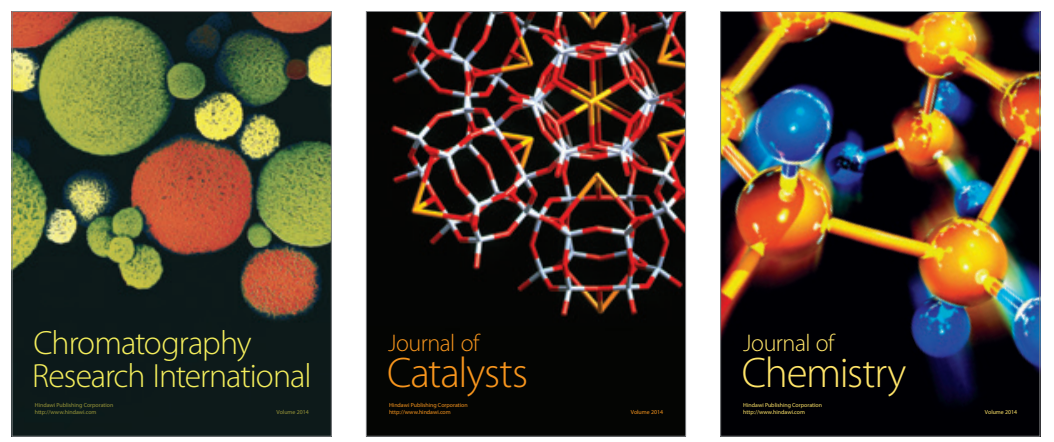
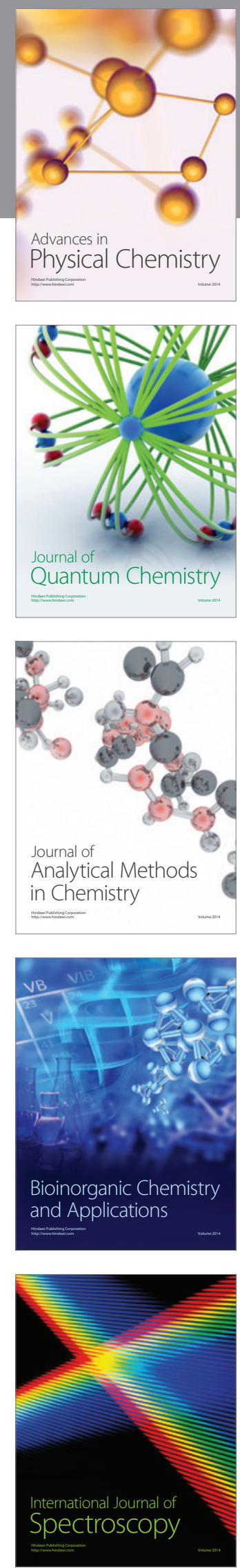